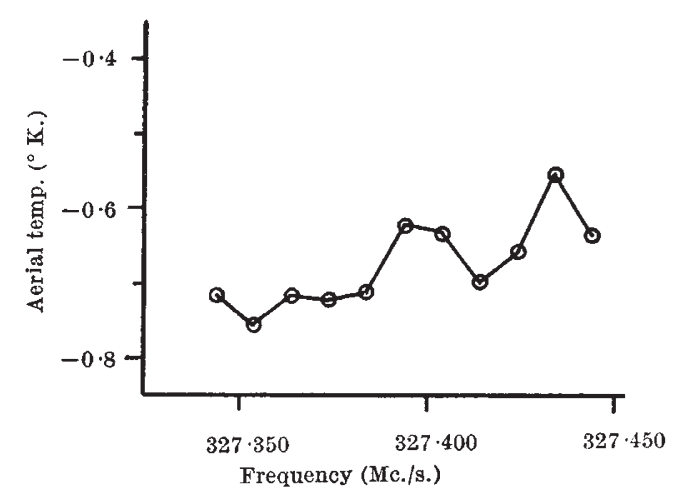

Fig. 1. Average of 14 days observations, uncorrected for receiver noise spectrum

the days because of disturbances attributable to interference, the remaining observations were corrected for Doppler frequency shift due to terrestrial motion. The average of the results for fourteen days is shown in Fig. 1, the root mean square value of the fluctuations corresponding to an aerial temperature of about $0.05^{\circ} \mathrm{K}$. The large mean displacement of about $-0.7 \mathrm{deg}$. K. is caused by the curvature in the spectrum of the receiver noise. To remove this dis. placement, control records were obtained $2 \mathrm{hr}$. before and $2 \mathrm{hr}$. after the observational run, with the aerial set respectively in the mean direction of the first and second halves of the run. This also helped to remove any part of the displacement which might be due to persistent low-level interference.

The final result is shown in Fig. 2, together with the expected ideal profile for a deuterium absorption intensity equivalent to an aerial temperature of $0.1^{\circ} \mathrm{K}$. and $30 \mathrm{kc} . / \mathrm{s}$. line-width. The aerial tomperature deduced from the observations has a mean value of about $-0.05^{\circ} \mathrm{K}$. Although this could be taken to indicate absorption in the region of search frequency, there are alternative explanations. It could be caused by weak terrestrial interference received predominantly on one or both reference frequencies; or it might be due to the different intensity relationships of the continuous background

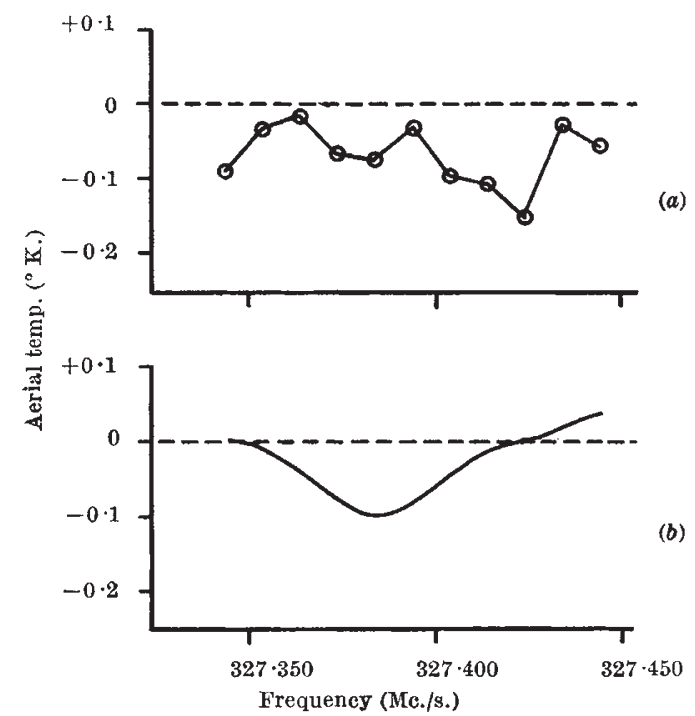

Fig. 2. (a) Corrected average of 14 days observations. (b) Expected proflie for a line intensity corresponding to $-0 \cdot 1^{\circ} \mathrm{K}$. aerial temperature radiation during the control period. Despite these uncertainties, it is reasonable to set $0.1^{\circ} \mathrm{K}$. as the upper limit of the intensity of deuterium absorption. These results represent an improvement in setting the lower limit of detection of deuterium absorption, for although Stanley and Prices with their larger aerial received about five times as much power from the continuous radiation, our temperature sensitivity is improved by a factor of 10 .

The precise derivation of the abundance of deuterium from the intensity of absorption is complex, since the continuous galactic radiation originates in several different ways. The principal sources of high brightness temperature are the narrow belt of emission parallel to the galactic equator which is largely associated with $\mathrm{H}$ II regions ${ }^{6}$, and a discrete source which is in the same direction but probably less distant than the galactic centre ${ }^{7}$; in addition, there is more widely diffused radiation of lower intensity. An approximate computation from our results leads to the conclusion that the average abundance of deuterium does not exceed $1 / 2,000$ that of hydrogen.

Acknowledgment is made to V. A. Hughes for helpful discussion and assistance in making observations.

R. L. Adgie

J. S. HEY

Radar Research Establishment, Ministry of Supply,

Great Malvern, Worcs. Dec. 14.

1 Shklovsky, I. S., Astro. J., U.S.S.R., \&9, 144 (1952).

"Getmanzev, G. G., Stankevitch, K. S., and Troitsky, V. S., Dok. Akad. Nauk, U.S.S.R., 103, 783 (1955).

${ }^{3}$ Suess, H. E., and Urey, H. C., Rev. Mot. Phys., 28, 53 (1956).

${ }^{4}$ Smith, R. A., Proc. Roy. Soc., A, 235, 1 (1956).

s Stanley, G. J., and Price, R., Nature, 177, 1221 (1956).

- Scheuer, P. A. G., and Ryle, M., Mon. Not. Roy. Astro. Soc., 113, 3 (1053).

7 McClain, E. F., Astrophys. J., 122, 376 (1955).

\section{Relation of II-Metre Solar System Phenomena to Solar Disturbances}

Three of the most important solar disturbances observed during 1956 were the great eruptive prominence of February 10 and the large flares of May 17 and August 31. For a week or two after each of these events, a sequence of phenomena involving Venus, the Earth and the Moon were observed with a radio telescope operating on a wave-length of $11 \mathrm{~m}$. ${ }^{1}$, these events appearing to be related to the preceding solar disturbance. In one case strong signals of apparent Venusian origin were observed a number of days after the solar disturbance, and a few days later a phenomenon was observed which, presumably, was caused by the presence of a large ion cloud near the Moon. Such a sequence of events suggests that a stream of particles ejected by the sun and travelling earthward first encountered the planet Venus, result. ing directly or indirectly in the production of strong 11-m. radio signals from Venus. Drifting farther out, the particles reached the vicinity of the Earth, with some of the particles forming a cloud near the Moon.

On February 10, 1956, there was a large solar prominence, which shot off the solar disk at very high velocity ${ }^{2}$. On February 18 a distinct interferometer pattern was obtained at the time of transit of the Moon through the antenna beam. The maxima of the pattern were characterized by many short static-like bursts of fractional second duration. 


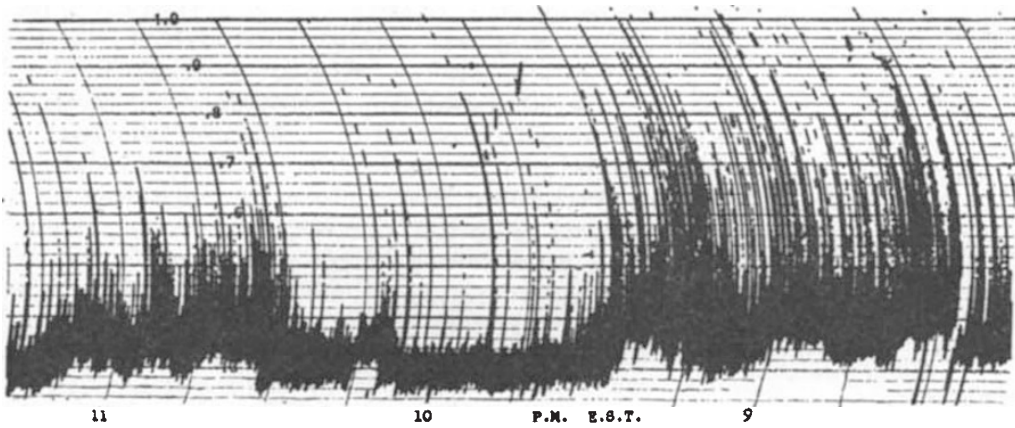

Fig. 1. 11-m. record obtained on September 16, 1956, during the transit of the Moon through the antenna beam

On May 17, 1956, there was a large solar flare rated as of importance 3 by the High Altitude Observatory $^{3}$, Boulder, Colorado. The events which followed were :

\begin{tabular}{|c|c|c|}
\hline Date & Event & Time after first event \\
\hline May 23 & Strong Class I Venus signals & 6 days \\
\hline May 24 & Strong Cygnus A scintillations & 7 days \\
\hline May 30 & Strong Class I Venus signals & 13 days \\
\hline June 1 & Strong Cygnus A scintillations & 15 days \\
\hline June 4,5 & $\begin{array}{l}\text { Moon reflexions of Class II Venus } \\
\text { signals }\end{array}$ & 18,19 days \\
\hline June 5 & Moon signals & 19 days \\
\hline
\end{tabular}

On August 31 there was another solar flare of importance 3 and on September 16 a record (Fig. 1) was obtained during the transit of the Moon through the antenna beam. It is characterized by an elevated background-level with strong static-like bursts of duration of a fraction of a second superposed. The brevity and intensity of these bursts are indicated by the dashed and dotted appearance of many of the bursts caused by the fact that the recorder pen was deflected so quickly as to skip over parts of the chart. Patterns similar to Fig. 1 were also obtained for about four days prior to September 16, the indicated time of transit for the source being about $50 \mathrm{~min}$. earlier on each preceding day. This observed motion corresponds closely to that of the Moon.

Referring to Fig. 1, the Moon was on the meridian at 10.12 p.m., E.S.T., and in the main beam from about 8.30 to 11.30 p.m. The interferometer pattern has three large lobes near 9 p.m. and a couple of smaller ones near 10.45 p.m. but with a very weak pattern between. Around 9 p.m. the background level is raised not only at the lobe maxima but also at the lobe minima. The magnitude of this effect indicates that the source must have been of the order of $1^{\circ}-2^{\circ}$ in angular extent. Furthermore, the nulls of the pattern are all displaced a number of minutes in right ascension from where they should be if the Moon itself were the source. To explain these effects we may postulate that associated with the Moon there was a cloud of charged particles, several times the angular extent of the Moon, with the radio centre of the cloud situated asymmetrically with respect to the Moon, being displaced at least $1^{\circ}$ toward smaller right ascension. It is not certain whether this cloud is itself the source of the $11 \cdot \mathrm{m}$. radiation, or whether the cloud acts as a huge reflecting surface which deflects $11-\mathrm{m}$. signals originating elsewhere, as on Venus, Jupiter or the Sun, all of which were below the horizon at the time. Although it is not clear what the primary source of the signals may be, it is convenient to refer to these radiations as 'Moon signals'.

As already reported", Moon reflexions were noted on June 4 and 5 of Class II signals, presumably of Venusian origin. However, as was mentioned, the observed difference in time between a direct Venusian pulse and its Moon echo was 6-10 per cent greater than the time calculated assuming the wave velocity of vacuum, and that the Moon itself was the reflecting object. If the Moon at this time had near it a large cloud of charged particles, as now seems likely, this time difference could be explained in several ways. Thus the particle cloud, acting as a conducting, dispersive medium, reduced the group velocity, or the cloud itself, displaced somewhat from the Moon but moving with it, acted as the reflecting surface, or both effects were present.

JoHn D. KraUS

Radio Observatory,

Ohio State University,

Columbus, Ohio. Nov. 14.

${ }^{2}$ Kraus, J. D., Nature, 178, 33 and 103 (1956).

${ }^{2}$ Menzel, D. H., et al., Astron. J., 61, 186 (1956).

${ }^{3}$ High Aititude Observatory and National Bureau of Standards, Boulder, Colorado, Preliminary Reports of Solar Activity.

${ }^{4}$ Kraus, J. D., Nature, 178, 159 (1956).

\section{Measurement of Ionospheric Path-Phase for Oblique Incidence}

THE observation of amplitudes of pulses received via various propagating modes has long been a standard technique, but observation of the corresponding carrier phases is more difficult. Several previous investigations have been made of the dynamic behaviour of the phase of the signal received in nearly vertical ionosphere reflexions, using $0 \cdot 15$ and $2 \cdot 4 \mathrm{Mc} . \mathrm{s}$. pulse transmissions ${ }^{1}$. Studies of the phase of a continuous carrier received at long ranges have beon made at $16 \mathrm{kc} . / \mathrm{s}$. and $a t^{3} 5 \mathrm{Mc} / \mathrm{s}$. Apparently, only Trautman, and Brennan and Phillips, of this Laboratory (private communication) have obtained results on the phase behaviour of individual oblique-incidence paths, using overlapping-pulse techniques in which the carrier phase of a given mode appears indirectly. The present communication describes three techniques, believed to be new, for direct measurement of changes of path-phase at oblique incidence, with a short sample record of each.

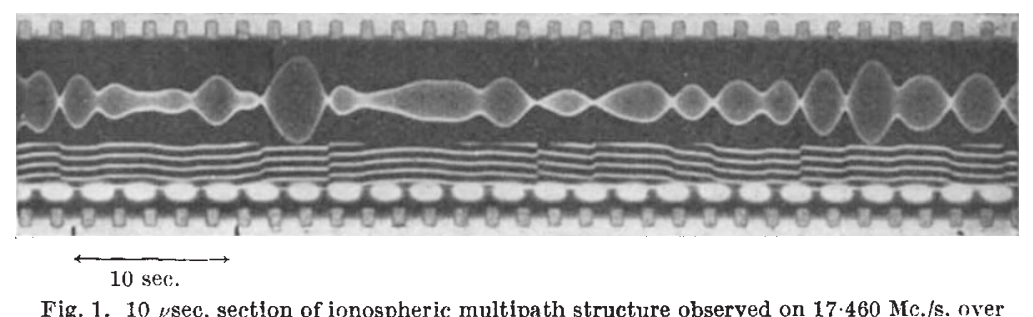

Fig. 1. $10 \mu$ sec. section of ionospheric multipath structure observed on $17.460 \mathrm{Mc}$. $/ \mathrm{s}$. over $4,100 \mathrm{~km}$. distance at $1660 \mathrm{E}$.S.T., July 21,1955 . Pulse-width about $160 \mu \mathrm{sec}$., repetitionminima. $A$ gradual change of frequency is also evidenced by the change in slope (method I) 